\title{
EULAR recommendations for the management of antiphospholipid syndrome in adults
}

\author{
Maria G Tektonidou, ${ }^{\oplus 1}$ Laura Andreoli, ${ }^{\circ}$ Marteen Limper, $^{3}$ Zahir Amoura, $^{4}$ \\ Ricard Cervera, ${ }^{5}$ Nathalie Costedoat-Chalumeau, ${ }^{\oplus 6}$ Maria Jose Cuadrado, ${ }^{7}$ \\ Thomas Dörner, ${ }^{\oplus 8}$ Raquel Ferrer-Oliveras, ${ }^{9}$ Karen Hambly, ${ }^{10}$ Munther A Khamashta, ${ }^{11}$ \\ Judith King, ${ }^{12}$ Francesca Marchiori, ${ }^{13}$ Pier Luigi Meroni, ${ }^{14}$ Marta Mosca, ${ }^{15}$ \\ Vittorio Pengo, ${ }^{16}$ Luigi Raio, ${ }^{17}$ Guillermo Ruiz-Irastorza, ${ }^{18}$ Yehuda Shoenfeld, ${ }^{19}$ \\ Ljudmila Stojanovich, ${ }^{20}$ Elisabet Svenungsson, ${ }^{21}$ Denis Wahl, ${ }^{22}$ Angela Tincani, ${ }^{2}$ \\ Michael M Ward ${ }^{\circledR 3}$
}

\section{Handling editor Josef $S$ Smolen \\ For numbered affiliations see end of article.}

\section{Correspondence to} Prof Maria G Tektonidou, First Department of Propaedeutic Internal Medicine, University of Athens, Athens 11527, Greece; mtektonidou@gmail.com

AT and MMW contributed equally.

Received 9 February 2019

Revised 16 April 2019

Accepted 18 April 2019

Published Online First

15 May 2019

Check for updates

(C) Author(s) (or their employer(s)) 2019. No commercial re-use. See rights and permissions. Published by BMJ.

To cite: Tektonidou MG,

Andreoli L, Limper M,

et al. Ann Rheum Dis

2019:78:1296-1304.

\section{ABSTRACT}

The objective was to develop evidence-based recommendations for the management of antiphospholipid syndrome (APS) in adults. Based on evidence from a systematic literature review and expert opinion, overarching principles and recommendations were formulated and voted. High-risk antiphospholipid antibody (aPL) profile is associated with greater risk for thrombotic and obstetric APS. Risk modification includes screening for and management of cardiovascular and venous thrombosis risk factors, patient education about treatment adherence, and lifestyle counselling. Lowdose aspirin (LDA) is recommended for asymptomatic aPL carriers, patients with systemic lupus erythematosus without prior thrombotic or obstetric APS, and nonpregnant women with a history of obstetric APS only, all with high-risk aPL profiles. Patients with APS and first unprovoked venous thrombosis should receive long-term treatment with vitamin $\mathrm{K}$ antagonists (VKA) with a target international normalised ratio (INR) of 2-3. In patients with APS with first arterial thrombosis, treatment with VKA with INR 2-3 or INR 3-4 is recommended, considering the individual's bleeding/ thrombosis risk. Rivaroxaban should not be used in patients with APS with triple aPL positivity. For patients with recurrent arterial or venous thrombosis despite adequate treatment, addition of LDA, increase of INR target to 3-4 or switch to low molecular weight heparin may be considered. In women with prior obstetric APS, combination treatment with LDA and prophylactic dosage heparin during pregnancy is recommended. In patients with recurrent pregnancy complications, increase of heparin to therapeutic dose, addition of hydroxychloroquine or addition of low-dose prednisolone in the first trimester may be considered. These recommendations aim to guide treatment in adults with APS. High-quality evidence is limited, indicating a need for more research.

\section{INTRODUCTION}

Antiphospholipid syndrome (APS) is a systemic autoimmune disorder with a wide range of vascular and obstetric manifestations associated with thrombotic and inflammatory mechanisms orchestrated by antiphospholipid (aPL) antibodies. Common APS clinical features include venous thromboembolism, stroke, recurrent early miscarriages and late pregnancy losses. ${ }^{1}$ According to current laboratory criteria for APS, aPL antibodies can be one of three types: lupus anticoagulant, anticardiolipin antibodies or antibeta2 glycoprotein I antibodies. Definite APS, fulfilling at least one clinical and one laboratory criteria of the updated Sapporo classification criteria, can occur in association with other autoimmune diseases, mainly systemic lupus erythematosus (SLE), or in its primary form (primary APS). ${ }^{12}$ Rarely, a life-threatening form of multiorgan thrombosis, known as catastrophic APS (CAPS), can occur. The presence of aPL in asymptomatic individuals or patients with SLE does not confirm the diagnosis of APS but can be associated with increased risk of thrombosis or pregnancy morbidity, depending on aPL characteristics and coexistence of other risk factors. ${ }^{34}$ The aPL type, the presence of multiple (double or triple) versus single aPL type, their titre (moderate-high titre vs low) and the persistence of aPL positivity in repeated measurements are defined as the 'aPL profile'. The aPL profile is an important factor determining the risk of thrombotic and obstetric events, and consequently the intensity of treatment. ${ }^{34}$

Clinical practice in APS is highly variable, in part because it is a rare disorder, and because knowledge about its diagnosis/classification, clinical spectrum and management is continuously advancing. There is a great heterogeneity among studies on the laboratory and clinical criteria used to define APS and the treatment approaches used over the past four decades. These factors make it often difficult to know the best approach to apply in daily practice. In addition, there is a paucity of high-quality randomised controlled trials (RCTs) in APS because of the difficulties in conducting adequately sized trials in an uncommon disease and using randomised designs among patients with often devastating clinical presentations. The objective of this project was to develop evidence-based recommendations for the prevention and management of adult APS that will help guide practice and improve quality of care and patient outcomes.

\section{METHODS}

We followed the updated European League Against Rheumatism (EULAR) standardised operating 
procedure $^{5}$ and used the Appraisal of Guidelines for Research \& Evaluation II tool. ${ }^{6}$ The steering committee included the convenor (MGT), co-convenor (AT), methodologist (MMW) and two physicians (LA, ML) responsible for the systematic literature reviews (SLRs) (both were members of the Emerging EULAR Network -(EMEUNET)). The task force included members from 11 European countries: 12 specialists in rheumatology or internal medicine, 2 obstetricians (RF-O, LR), 2 physicians from vascular medicine/thrombosis centres (VP, DW), 1 healthcare professional (KH), and 2 patient representatives (FM, JK).

The convenor prepared the first task force meeting and the first set of research questions on four major topics: risk stratification and risk modification in asymptomatic aPL-positive patients, primary and secondary thromboprophylaxis in APS, management of obstetric APS, and management of CAPS. The research questions were discussed among the task force members, and a set of 31 research questions was formulated using the PICO format (P, population; I, intervention; C, comparator; $\mathrm{O}$, outcomes) and voted according to the Delphi method at a meeting in December 2017.

The data sources for the SLR were PubMed, Embase and the Cochrane Library, which were searched for relevant English-language published articles from their inceptions through 31 January 2018. We included one RCT published after this date because of the importance of its reported safety results (Trial on Rivaroxaban in Antiphospholipid Syndrome (TRAPS) trial). ${ }^{7}$ Search terms were developed with the aid of an experienced librarian, who performed the searches. All titles and abstracts of retrieved articles were first reviewed independently by the two literature reviewers. The full-text articles were then reviewed independently by three persons: one literature reviewer, convenor and methodologist. The data abstraction was performed by the two literature reviewers according to a written protocol and supervised by the methodologist. Data abstraction was then independently double-checked by the convenor and methodologist. Each included article was graded for its methodological quality and assigned to the relevant PICO question by the convenor and methodologist. Data were tabulated and summarised by the methodologist in an evidence report that included summary of findings tables with pooled estimates of effect sizes for studies that directly addressed the population, intervention and comparator of PICO questions. Based on this evidence, a first draft of recommendations on 12 topics was prepared by the convenor and co-convenor. The draft recommendations and evidence report were sent to all task force members for review prior to the second task force meeting.

The second task force meeting, held on 3 September 2018, included the presentation of SLR results, discussions of the first draft of recommendations in four working groups, working group presentations of the edited draft for each topic, and discussion and voting of the text. Incorporating additional discussions on wording changes, the final set of overarching principles and recommendations, including the level of evidence (LoE) and grade of recommendations (GoR) according to the Oxford Centre for Evidence-Based Medicine standards ${ }^{8}$ prepared by the methodologist in collaboration with convenors, was sent by the convenor to all task force members, who voted anonymously on the level of agreement (LoA). A rating scale of $0-10$ (0: do not agree; 10: fully agree) was used for each recommendation. The manuscript was reviewed and approved by all task force members and the EULAR Executive Committee before submission.
Box 1 Definitions of medium-high antiphospholipid antibody (aPL) titres, and of high-risk and low-risk aPL profile

Medium-high aPL titres.

- Anticardiolipin ( $\mathrm{aCL}$ ) antibody of $\lg \mathrm{G}$ and/or IgM isotype in serum or plasma present in titres $>40 \mathrm{lgG}$ phospholipid (GPL) units or $>40$ IgM phospholipid (MPL) units, or $>$ the 99th percentile, measured by a standardised ELISA. Antibeta2 glycoprotein I antibody of $\lg G$ and/or IgM isotype in serum or plasma in titre $>$ the 99 th percentile, measured by a standardised ELISA. ${ }^{1}$

High-risk aPL profile.

- The presence (in 2 or more occasions at least 12 weeks apart) of lupus anticoagulant (measured according to ISTH guidelines), or of double (any combination of lupus anticoagulant, aCL antibodies or antibeta2 glycoprotein I antibodies) or triple (all three subtypes) aPL positivity, or the presence of persistently high aPL titres.

Low-risk aPL profile.

- Isolated aCL or antibeta2 glycoprotein I antibodies at lowmedium titres, particularly if transiently positive. ${ }^{3}$

\section{RESULTS}

The SLR yielded 7534 articles and 15 hand-searched articles. After the titles and abstracts review, 670 articles were selected for full-text review. The full-text review yielded 249 articles for data abstraction; 61 were excluded and 188 articles were finally considered to be relevant. A detailed presentation of the results of the SLRs that informed the task force recommendations is published separately and should be reviewed together with this report. ${ }^{9}$ Based on the SLR results and expert opinion, 3 overarching principles and 12 recommendations were developed.

\section{Overarching principles}

1. Identifying the presence of factors associated with high risk for thrombotic and obstetric events is critical in patient management. A major risk factor is the high-risk aPL profile, including any of the following: the presence of lupus anticoagulant as the aPL subtype most closely related to thrombosis, the presence of double (any combination of lupus anticoagulant, anticardiolipin antibodies or antibeta2 glycoprotein I antibodies) or triple (all three subtypes) aPL positivity, or the presence of persistently high aPL titres, ${ }^{410}$ as also shown by the aPL score ${ }^{11}$ and Global Anti-Phospholipid Syndrome (GAPSS) Score. ${ }^{12}$ Definitions of medium-high aPL titres and of high-risk and low-risk aPL profiles are included in box 1. Additional risk factors for clinical events are coexistence of other systemic autoimmune diseases, especially SLE, a history of thrombotic and/or obstetric APS, and the presence of traditional cardiovascular risk factors. ${ }^{3410}$

2. The guidelines for cardiovascular disease (CVD) prevention in the general population should be followed. Screening for and management of venous thrombosis risk factors are also recommended. Heparin at prophylactic dosage, preferably low molecular weight heparin (LMWH), should be used in high-risk situations such as surgery, prolonged immobilisation and the puerperium.

3. All patients treated with vitamin $\mathrm{K}$ antagonists (VKA) should receive counselling about treatment adherence, the need for 
close international normalised ratio (INR) monitoring especially in the setting of newly initiated treatment or bridging with heparin, the protocol of perioperative bridging therapy with heparin, and drug and food interactions. Counselling should be provided on the use of contraceptives, pregnancy planning and postmenopausal hormone therapy for all women with APS. ${ }^{13}$ Patients should also receive dietary counselling for CVD prevention. Physical activity is encouraged in patients with APS including those on oral anticoagulants.

\section{RECOMMENDATIONS}

Table 1 presents the LoE, GoR and LoA for each recommendation. For recommendations with B GoR, we used the statement 'is recommended'. For C and D grades, we mostly used the terms 'may be considered' or 'could be considered', with some exceptions according to experts' judgement about the importance of the intervention. Recommendations that are phrased as 'is recommended' are those that the task force meant, based on the evidence and their experience, should be followed in almost all cases.

\section{Primary thromboprophylaxis in aPL-positive subjects}

1.In asymptomatic aPL carriers (not fulfilling any vascular or obstetric APS classification criteria) with a high-risk aPL profile with or without traditional risk factors, prophylactic treatment with low-dose aspirin (LDA) (75-100 mg daily) is recommended. Use of LDA for primary prophylaxis is supported by results of a meta-analysis of seven observational studies of 460 asymptomatic aPL carriers that found the risk of first thrombosis to be reduced by half in those who used LDA versus those who did not use LDA. ${ }^{14}$ Most patients had high-risk aPL profiles, but few had traditional CVD risk factors. An association of similar magnitude was present in a smaller individual patient meta-analysis derived from these studies. ${ }^{15}$ Neither meta-analyses display worrisome variations as the directions were clear. Although evidence was largely from observational studies, the panel recommended the use of LDA for primary prophylaxis in asymptomatic aPL individuals with high-risk profile given the likelihood of benefit and low risk of adverse events of this intervention.

2.In patients with SLE and no history of thrombosis or pregnancy complications:

A. With high-risk aPL profile, prophylactic treatment with LDA is recommended.

B. With low-risk aPL profile, prophylactic treatment with LDA may be considered.

Treatment with LDA for patients with SLE and high-risk aPL profile is supported by a subanalysis of eight studies, mostly observational, in a meta-analysis. ${ }^{14}$ In this analysis, risk of first thrombosis was reduced by almost half among patients treated with LDA versus patients not treated, without major bleeding events. In an individual patient analysis, this association was independent of the use of hydroxychloroquine (HCQ), suggesting that LDA offers additional benefit in this patient group. ${ }^{15}$ Patients with high-risk aPL profile comprised the majority (but not all) of patients in these studies. Although there was heterogeneity between the studies, the direction of effect was clear. Less evidence is available on the use of LDA in patients with SLE and low-risk aPL profile, but pooled data from two cohort studies indicate that the use of LDA was also associated with a lower risk of thrombosis in this group. ${ }^{16} 17$

3.In non-pregnant women with a history of obstetric APS only (with or without SLE), prophylactic treatment with LDA after adequate risk/benefit evaluation is recommended.
The primary prevention of thrombosis with LDA in women with a history of obstetric APS without SLE was addressed in a meta-analysis including five observational studies. ${ }^{14}$ The pooled OR for first thrombosis associated with use of LDA was 0.25 (95\% CI 0.10 to 0.62 ). Studies of women with SLE and prior obstetric APS are scarce, but the protective effect of LDA was supported by three retrospective studies that included a minority of patients with SLE. ${ }^{18-20}$ The panel recommended the use of LDA in women with a history of obstetric APS only, according to their thrombosis/bleeding risk (aPL profile, coexistent traditional cardiovascular risk factors, intolerance/contraindication to aspirin).

\section{Secondary thromboprophylaxis in APS}

4.In patients with definite APS and first venous thrombosis: A.Treatment with VKA with a target INR 2-3 is recommended. In patients with APS and first venous thrombosis, after an initial therapy with unfractionated heparin (UFH) or LMWH and bridging therapy of heparin plus VKA, treatment with VKA with a target INR of $2-3$ is recommended. Data from an $\mathrm{RCT}^{21}$ reporting exclusively on patients with venous events and pooled data from five studies that included a majority with venous events ${ }^{21-25}$ showed no additional benefit of a target INR of 3-4 vs INR of $2-3$. However, evidence is limited by the frequent failure to achieve the target INR in the high-intensity groups in the RCTs. Data on bleeding were not reported for patients with venous thrombosis specifically. However, although not based on data from these studies, higher level of anticoagulation would be anticipated to also have a higher risk of bleeding.

B.Rivaroxaban should not be used in patients with triple aPL positivity due to the high risk of recurrent events. Direct oral anticoagulants (DOACs) could be considered in patients not able to achieve a target INR despite good adherence to VKA or those with contraindications to VKA (eg, allergy or intolerance to VKA). Despite the broadening use of DOACs in secondary thrombosis prevention in the general population, there is limited evidence about their effectiveness and safety in APS. In a post-hoc analysis of patients with APS included in three RCTs of dabigatran versus warfarin, ${ }^{26}$ and in one RCT of rivaroxaban versus warfarin in patients with venous thrombotic APS, ${ }^{27}$ there were no differences in outcomes between treatment with DOACs and VKA for venous thrombosis, but the evidence is limited by small samples, under-representation of high-risk patients with APS and short follow-up. A recent RCT of rivaroxaban versus warfarin in patients with APS with triple aPL positivity was prematurely terminated due to an excess of thromboembolic events (mostly arterial) in the rivaroxaban arm. $^{7}$ Accordingly, rivaroxaban should not be used in patients with triple aPL positivity. The panel agreed that DOACs may be considered in patients with difficulty achieving a target INR of 2-3 despite compliance with VKA or who have contraindications to VKA. Switching from treatment with VKA to DOACs due to low adherence to VKA or INR monitoring should be avoided.

C.In patients with unprovoked first venous thrombosis, anticoagulation should be continued longterm.

Use of long-term anticoagulation in patients with APS is supported by two small direct comparison studies (one RCT, one retrospective cohort $)^{2829}$ that showed a lower risk of recurrent venous thrombosis among patients with APS on long-term vs 3-6 months of oral anticoagulation. However, studies did not specify the proportion of patients with unprovoked thrombosis, making this evidence indirect. 
Table 1 EULAR recommendations for the prevention and management of APS in adults

\section{Overarching principles}

1. Risk stratification in aPL-positive individuals should include determination of the presence of a high-risk aPL profile (defined as any of the following: multiple aPL positivity, lupus anticoagulant or persistently high aPL titres), history of thrombotic and/or obstetric APS, coexistence of other systemic autoimmune diseases such as SLE, and the presence of traditional cardiovascular risk factors.

2. General measures for aPL-positive individuals should include screening for and strict control of cardiovascular risk factors (smoking cessation; management of hypertension, dyslipidaemia and diabetes; and regular physical activity) in all individuals and particularly those with a high-risk aPL profile, screening for and management of venous thrombosis risk factors, and use of LMWH in high-risk situations such as surgery, hospitalisation, prolonged immobilisation and the puerperium.

3. Patient education and counselling on treatment adherence, INR monitoring in patients treated with VKA, use of perioperative bridging therapy with LMWH for patients on oral anticoagulants, oral contraceptive use, pregnancy and postpartum period, postmenopausal hormone therapy, and lifestyle recommendations (diet, exercise) are important in the management of APS.

Recommendations

\section{Statement, LoE*/GoRt}

LoA (0-10)‡

Primary thromboprophylaxis in aPL-positive subjects

1. In asymptomatic aPL carriers (not fulfilling any vascular or obstetric APS classification criteria) with a high-risk aPL profile with or without traditional risk factors, prophylactic treatment with LDA (75-100 mg daily) is recommended (2a/B).

2. In patients with SLE and no history of thrombosis or pregnancy complications:

A. With high-risk aPL profile, prophylactic treatment with LDA is recommended (2a/B).

B. With low-risk aPL profile, prophylactic treatment with LDA may be considered (2b/C).

3. In non-pregnant women with a history of obstetric APS only (with or without SLE), prophylactic treatment with LDA after adequate risk/benefit evaluation is recommended (2b/B).

Secondary thromboprophylaxis in APS

4. In patients with definite APS and first venous thrombosis:

A. Treatment with VKA with a target INR 2-3 is recommended (1b/B).

B. Rivaroxaban should not be used in patients with triple aPL positivity due to the high risk of recurrent events (1b/B). DOACs could be considered in patients not able to achieve a target INR despite good adherence to VKA or those with contraindications to VKA (eg, allergy or intolerance to VKA) (5/D).

C. In patients with unprovoked first venous thrombosis, anticoagulation should be continued long term (2b/B).

D. In patients with provoked first venous thrombosis, therapy should be continued for a duration recommended for patients without APS according to international guidelines (5/D). Longer anticoagulation could be considered in patients with high-risk aPL profile in repeated measurements or other risk factors for recurrence (5/D).

5. In patients with definite APS and recurrent venous thrombosis despite treatment with VKA with target INR of 2-3:

A. Investigation of, and education on, adherence to VKA treatment, along with frequent INR testing, should be considered (5/D).

B. If the target INR of 2-3 had been achieved, addition of LDA, increase of INR target to 3-4 or change to LMWH may be considered (4-5/D).

6. In patients with definite APS and first arterial thrombosis:

A. Treatment with VKA is recommended over treatment with LDA only (2b/C).

B. Treatment with VKA with INR 2-3 or INR 3-4 is recommended, considering the individual's risk of bleeding and recurrent thrombosis (1 b/B). Treatment with VKA with INR 2-3 plus LDA may also be considered (4/C).

C. Rivaroxaban should not be used in patients with triple aPL positivity and arterial events (1b/B). Based on the current evidence, we do not recommend use of DOACs in patients with definite APS and arterial events due to the high risk of recurrent thrombosis (5/D).

7. In patients with recurrent arterial thrombosis despite adequate treatment with VKA, after evaluating for other potential causes, an increase of INR target to 3-4, addition of LDA or switch to LMWH can be considered (4-5/D).

Obstetric APS

8. In women with a high-risk aPL profile but no history of thrombosis or pregnancy complications (with or without SLE), treatment with LDA (75-100 mg daily) during pregnancy should be considered (5/D).

9. In women with a history of obstetric APS only (no prior thrombotic events), with or without SLE: A. With a history of $\geq 3$ recurrent spontaneous miscarriages $<10$ th week of gestation and in those with a history of fetal loss ( $\geq 10$ th week of gestation), combination treatment with LDA and heparin at prophylactic dosage during pregnancy is recommended ( $2 \mathrm{~b} / \mathrm{B})$.

B. With a history of delivery $<34$ weeks of gestation due to eclampsia or severe pre-eclampsia or due to recognised features of placental insufficiency, treatment with LDA or LDA and heparin at prophylactic dosage is recommended considering the individual's risk profile $(2 \mathrm{~b} / \mathrm{B})$.

C. With clinical 'non-criteria' obstetric APS such as a the presence of two recurrent spontaneous miscarriages $<10$ th week of gestation, or delivery $\geq 34$ weeks of gestation due to severe pre-eclampsia or eclampsia, treatment with LDA alone or in combination with heparin might be considered based on the

individual's risk profile (4/D). 
Table 1 Continued

Recommendations

D. With obstetric APS treated with prophylactic dose heparin during pregnancy, continuation of heparin at

prophylactic dose for 6 weeks after delivery should be considered to reduce the risk of maternal thrombosis $(4 / C)$.

10. In women with 'criteria' obstetric APS with recurrent pregnancy complications despite combination treatment with LDA and heparin at prophylactic dosage, increasing heparin dose to therapeutic dose (5/D) or addition of HCQ (4/D) or low-dose prednisolone in the first trimester (4/D) may be considered. Use of intravenous immunoglobulin might be considered in highly selected cases (5/D).

11. In women with a history of thrombotic APS, combination treatment of LDA and heparin at therapeutic dosage during pregnancy is recommended (4/C).

CAPS

12. A. Prompt treatment of infections by early use of anti-infective medications in all aPL-positive individuals and minimisation of interruptions in anticoagulation or low INR level in patients with thrombotic APS are recommended to help prevent the development of CAPS (4/D).

B. For first-line treatment of patients with CAPS, combination therapy with glucocorticoids, heparin and plasma exchange or intravenous immunoglobulins is recommended over single agents or other combinations of therapies. Additionally, any triggering factor (eg, infections, gangrene or malignancy) should be treated accordingly (5/D).

In patients with refractory CAPS, B cell depletion (eg, rituximab) or complement inhibition (eg, eculizumab) therapies may be considered (4/D).

When there are multiple ratings, these follow the order of corresponding subpart of the recommendation.

* Level of evidence (LoE): 1a: systematic review of RCTs; 1b: individual RCT; 2a: systematic review of cohort studies; 2b: individual cohort study (and low-quality RCT); 3a: systematic review of case-control studies; 3b: individual case-control study; 4: case series and poor-quality cohort and case-control studies; 5 : expert opinion without explicit critical appraisal, or based on physiology, bench research or 'first principles'.

tGrade of recommendation (GoR): A: consistent level 1 studies; B: consistent level 2 or 3 studies, or extrapolations from level 1 studies; C: level 4 studies or extrapolations from level 2 or 3 studies; D: level 5 evidence or troublingly inconsistent or inconclusive studies of any level.

¥Numbers in column 'LoA' indicate the mean and SD (in parenthesis) of the level of agreement among task force members.

APS, antiphospholipid syndrome; aPL, antiphospholipid antibodies. CAPS, catastrophic APS; DOACs, direct oral anticoagulants; EULAR, European League Against Rheumatism; HCQ, hydroxychloroquine; INR, international normalised ratio; LDA, low-dose aspirin; LMWH, low molecular weight heparin; LoA, level of agreement; RCT, randomised controlled trial;

SLE, systemic lupus erythematosus; VKA, vitamin K antagonists;

D.In patients with provoked first venous thrombosis, therapy should be continued for a duration recommended for patients without APS according to international guidelines. Longer anticoagulation could be considered in patients with high-risk aPL profile in repeated measurements or other risk factors for recurrence.

This recommendation was based on expert opinion because we did not identify any studies that directly addressed the question of treatment duration after the initial provoked venous thrombosis. The panel recommended a duration of anticoagulation according to international guidelines for patients without $\mathrm{APS}^{30}$ because the benefit of long-term anticoagulation in this population is unclear. In patients with repeatedly high-risk aPL profile or those with additional risk factors for thrombosis recurrence, longer anticoagulation may be considered.

5. In patients with definite APS and recurrent venous thrombosis despite treatment with VKA with a target INR of 2-3:

A. Investigation of, and education on, adherence to VKA treatment, along with frequent INR testing, should be considered.

B. If the target INR of 2-3 had been achieved, addition of LDA, increase of INR target to 3-4 or change to LMWH may be considered.

There is limited evidence, mainly from case series, about therapeutic strategies for patients who have recurrent venous thrombosis despite a target INR of 2-3. Evaluation of the intensity of anticoagulation and adherence to treatment, patient counselling, frequent INR monitoring or a self-monitoring programme are important in optimising anticoagulation management. For adherent patients who have a recurrent thrombotic event, the addition of LDA, increase of INR target to 3-4 or switch to LMWH can be considered based on the individual's characteristics and preferences (aspirin intolerance/contraindication, cost and side effects of continuous LMWH use). There is insufficient evidence to determine the relative efficacy and safety of these options in this patient group.

6. In patients with definite APS and first arterial thrombosis:

A.Treatment with VKA is recommended over treatment with LDA only.

The use of VKA over LDA is supported by data from observational studies that showed a lower likelihood of recurrent thrombosis among patients with APS and prior arterial thrombosis (mainly stroke) treated with VKA versus LDA alone. ${ }^{31} 32$ An early study in older patients with strokes reported no difference in event recurrences between LDA and warfarin, but aPL was tested only once and was mainly of low titre in this study. ${ }^{33}$ These issues make it difficult to apply the latter results to patients of any age who fulfil the laboratory criteria for APS.

B.Treatment with VKA with INR 2-3 or INR 3-4 is recommended, considering the individual's risk of bleeding and recurrent thrombosis. Treatment with VKA with INR 2-3 plus LDA may also be considered.

Patients with APS with arterial thrombosis have a higher risk of recurrence compared with those with venous thrombosis, and a tendency for recurrences in the same vascular (arterial) bed. ${ }^{34}$ An earlier SLR including mainly observational studies reported that recurrent events occurred more often in patients with APS treated with VKA with a target INR of 2-3 compared with an INR of 3-4, but outcomes among patients with first arterial thrombosis were not analysed specifically. ${ }^{35}$ This review did not compare the target INR 2-3 and INR 3-4 arms within the same study, but disaggregated the arms. Pooled data from two retrospective studies and two $\mathrm{RCTs}^{21-24}$ showed that there was no statistically significant difference in thrombosis recurrences between treatment with VKA with a target INR of 3-4 and treatment with INR of 2-3 (relative risk (RR) 0.46 (0.06-3.52)). However, these studies included a mixture of patients with 
either venous or arterial thrombosis, and a minority had arterial events. In one trial that provided data specifically on patients with arterial thrombosis, there was no difference in recurrences between those treated to a target INR of $2-3$ or INR of 3-4 but without statistical significance (HR 3.1 (0.3-30.0)), although the sample was small and the achievement of a target INR of 3-4 was low. ${ }^{21}$ Because of these limitations, the higher intensity INR approach is preferred by some centres. In decision-making, physicians should take into account the individual's risk of recurrent thrombosis and major bleeding, as well as the patient's preferences after discussion. Alternatively, treatment with VKA with a target INR of 2-3 plus LDA is used by some experts, supported by limited data from one retrospective cohort study and one small RCT. ${ }^{3637}$

C.Rivaroxaban should not be used in patients with triple aPL positivity and arterial events. Based on the current evidence, we do not recommend use of DOACs in patients with definite APS and arterial events due to the high risk of recurrent thrombosis.

According to the results of the TRAPS trial, ${ }^{7}$ rivaroxaban should not be used in triple aPL-positive patients with APS. In addition, an ongoing trial of apixaban in APS (Apixaban for the Secondary Prevention of Thromboembolism among patients with the AntiphosPholipid Syndrome ((ASTRO-APS)) (ClinicalTrials.gov identifier: NCT02295475) was recently modified after evaluation of their initial data to exclude patients with arterial thrombosis. Based on these data and those from case series reporting arterial thrombosis recurrences in patients with APS treated with DOACs, use of DOACs is not currently recommended in patients with definite APS and arterial events. ${ }^{38}$ Ongoing clinical trials will help to better define the role of DOACs in APS.

7.In patients with recurrent arterial thrombosis despite adequate treatment with VKA, after evaluating for other potential causes, an increase of INR target to 3-4, addition of LDA or switch to LMWH can be considered.

Evidence on the management of recurrent arterial thrombosis despite VKA treatment is limited. The panel agreed that after evaluating other risk factors for thrombosis (eg, traditional cardiovascular risk factors, cancer, other thrombophilic states) and investigating the adherence to VKA treatment, increase of target INR to 3-4, or INR 2-3 with the addition of LDA, or switching to LMWH may be considered. Adjunctive therapy with antimalarials or statins could also be considered. ${ }^{4} 10$ 39-41

\section{Obstetric APS}

8.In women with a high-risk aPL profile but no history of thrombosis or pregnancy complications (with or without SLE), treatment with LDA (75-100 mg/day) during pregnancy should be considered.

Data from one placebo-controlled RCT of LDA in six women with $\mathrm{SLE}^{42}$ and data from three low-quality studies (two RCTs, one retrospective cohort) ${ }^{43-45}$ of women without SLE found no difference in the prevalence of live births with use of LDA. However, these studies did not specifically include women with a high-risk aPL profile. The panel agreed that use of LDA should be considered in pregnant women with high-risk aPL profile due to the risk of obstetric and thrombotic complications during pregnancy associated with high-risk aPL profile.

9.In women with a history of obstetric APS only (no prior thrombotic events), with or without SLE:

A. With a history of $\geq 3$ recurrent spontaneous miscarriages $<10$ th week of gestation and in those with a history of fetal loss $(\geq 10$ th week of gestation), combination treatment with LDA and heparin at prophylactic dosage during pregnancy is recommended.
Pooled data from one RCT including only patients with a history of first trimester losses ${ }^{46}$ and eight supporting observational studies ${ }^{47-54}$ that did not exclusively study women with early pregnancy losses indicated a higher likelihood of live births with combination treatment with LDA and heparin at prophylactic dosage versus LDA alone. Some experts believe that LDA alone can be also effective. For women with a history of fetal loss, combination treatment with LDA and heparin was associated with a higher likelihood of live birth compared with treatment with LDA alone. However, these studies included women with histories of both early and mid-pregnancy losses. ${ }^{48-52}$ LDA should be preferably started prior to conception, and heparin (LMWH or UFH) should be added as soon as pregnancy is confirmed. LMWH is preferred for practical reasons.

B.With a history of delivery $<34$ th week of gestation due to eclampsia or severe pre-eclampsia or due to recognised features of placental insufficiency, treatment with LDA or LDA and heparin at prophylactic dosage is recommended considering the individual's risk profile.

Data from two studies (one RCT, one retrospective cohort) ${ }^{4855}$ showed that the likelihood of live births did not differ between women treated with LDA plus heparin and those treated with LDA alone. Physicians should tailor their treatment approach to the individual's risk assessment including aPL profile and other risk situations (eg, presence of other cardiovascular risk factors or immobility).

C. With clinical 'non-criteria' obstetric APS, treatment with LDA alone or in combination with heparin might be considered based on the individual's risk profile.

The 'non-criteria' obstetric APS manifestations included in our search were the presence of two recurrent spontaneous miscarriages $<10$ th week of gestation or delivery $\geq 34$ weeks of gestation due to severe pre-eclampsia or eclampsia. Because studies combined several types of pregnancy losses without specifying on the proportion of 'non-criteria' APS and due to very limited evidence, this recommendation is mainly based on expert opinion. Because of a potential higher risk for obstetric and/or thrombotic complications during pregnancy in women with a history of clinical 'non-criteria' obstetric APS, the panel agreed that treatment with LDA alone or in combination with heparin might be considered, based on an individual's risk profile (aPL profile, concomitant SLE, prior live births, and additional risk factors for pregnancy loss or thrombosis).

D. With obstetric APS treated with prophylactic dose heparin during pregnancy, continuation of heparin at prophylactic dose for 6 weeks after delivery should be considered to reduce the risk of maternal thrombosis.

No studies directly tested the efficacy of extending treatment with prophylactic heparin after delivery. The panel suggested that in women receiving prophylactic dose heparin during pregnancy, the same dosage of heparin should be continued for 6 weeks after delivery due to an increased risk of thrombosis at puerperium.

10.In women with 'criteria' obstetric APS with recurrent pregnancy complications despite combination treatment with LDA and heparin at prophylactic dosage, increasing heparin dose to therapeutic dose or addition of HCQ or low-dose prednisolone in the first trimester may be considered. Use of intravenous immunoglobulin (IVIG) might be considered in highly selected cases.

The most common practice if the combination of LDA and prophylactic dose heparin fails is to increase the dose of heparin to therapeutic dose, although no supporting evidence exists. Other treatment strategies may include the addition of HCQ or low prednisolone doses in the first trimester. Evidence directly 
supporting these treatment options is based on two small observational studies with limited representativeness. ${ }^{56} 57$ Use of IVIG was not associated with a higher proportion of live births compared with conventional treatment in three small observational studies ${ }^{58-60}$ that directly addressed this question, although confounding by indication may have occurred. Although the expectation of benefit is small, the panel agreed that IVIG might be considered in highly selected cases when other treatments have failed.

11.In women with a history of thrombotic APS, combination treatment of LDA and heparin at therapeutic dosage during pregnancy is recommended.

In observational studies, treatment with LDA and therapeutic dose heparin was associated with live births in $79 \%$ of pregnancies on average. ${ }^{52}$ B1-64 Because a history of thrombotic APS is associated with increased risk for future thrombotic or obstetric events, ${ }^{65}$ treatment with LDA and heparin at therapeutic dosage during pregnancy is recommended. Switching treatment from VKA to therapeutic dose LMWH or UFH is recommended as soon as pregnancy is confirmed, ideally before the sixth week of gestation due to the teratogenic effects of warfarin. ${ }^{66}$

\section{Catastrophic APS}

12. The most common precipitating factors for the development of CAPS are anticoagulation discontinuation among patients with prior diagnosis of APS, infections and surgical procedures. ${ }^{67}$ Early diagnosis and management of infections and minimisation of discontinuation or low-intensity anticoagulation, especially perioperatively, are recommended. ${ }^{68}$ Based on the recently published clinical practice guidelines for CAPS management, combination therapy with glucocorticoids, heparin and plasma exchange or IVIG is recommended over single agents as firstline treatment of patients with CAPS. ${ }^{69}$ Concurrent treatment of precipitating factors is also recommended (eg, infections, gangrene or malignancy). For refractory CAPS, B cell depletion (eg, rituximab) or complement inhibition (eg, eculizumab) therapies may be considered based on data from case reports. ${ }^{69}$

\section{DISCUSSION}

APS is a complex disorder and its management often involves collaboration among several medical specialties. The aim of these recommendations is to provide guidance to all health professionals involved in patient care, inform patients and support their engagement in shared decision-making, and provide evidence to researchers, funders and policy makers. The task force included members from several professional groups covering different perspectives and also involved two patients who participated actively in both meetings.

The main challenge in developing recommendations for the management of adult APS was the low certainty of evidence. Many studies included patients with a mix of different clinical features and did not provide stratified data for arterial or venous thrombosis separately or for each of various types of obstetric APS. This resulted in only indirect evidence for many of the treatment decisions that were examined. Several meta-analyses also pooled studies of heterogeneous patient groups. A high risk of bias and low power, mostly due to the rarity of the syndrome, were also common in RCTs. Therefore, a significant number of recommendations necessarily relied more, or only, on low-quality evidence or expert opinion. An important consideration for future research would be well-designed observational studies and RCTs of homogeneous patient populations. These studies will hopefully increase the quality of evidence for the
Box 2 Research agenda

Risk stratification.

- Better definition of high-risk and low-risk aPL profile. Better delineation of the risk associated with different aPL profiles to allow improved classification of patients in research studies.

Primary thrombosis prevention.

- Impact on thrombosis risk of intensive management of traditional risk factors such as smoking cessation, control of hypertension, dyslipidaemia and sedentary behaviour.

- Evaluation of the role of HCQ for primary thrombosis prevention in subjects with high-risk aPL profile: (1) asymptomatic aPL carriers, (2) patients with a history of obstetric APS without SLE and (3) non-criteria APS manifestations (eg, thrombocytopaenia, heart valve disease and aPL-associated nephropathy).

- Evaluation of the role of statins or coenzyme Q10 for primary thrombosis prevention.

\section{Secondary thrombosis prevention.}

- Controlled studies of the efficacy and safety of treatment with VKA with target INR of 3-4 versus combination treatment of VKA with target INR of 2-3 and LDA for patients with a history of first arterial thrombosis.

- Duration of VKA in provoked first venous thrombosis.

- Controlled studies of the efficacy of therapy of VKA alone versus VKA plus HCQ for patients with a history of first arterial thrombosis.

- Controlled studies of the efficacy and safety of targeted therapies (eg, B cell depletion therapy, complement inhibitors, or mammalian target of rapamycin (mTOR) inhibitors) in recurrent arterial thrombotic events despite treatment with VKA with a target INR of 3-4.

- Adjunctive treatment for recurrent arterial thrombosis: $\mathrm{HCQ}$, statins or vitamin D. Evaluation of the role of platelet inhibitors (other than LDA), for example, ADP receptor inhibitors, adenosine reuptake inhibitors and others.

- Discontinuation of VKA treatment in patients who became negative for $\mathrm{aPL}$ in repeated measurements.

\section{Obstetric APS.}

- Controlled studies of the efficacy and safety of treatment with LDA and heparin versus treatment with LDA, heparin and HCQ in women with a history of recurrent obstetric complications.

- Efficacy of $150 \mathrm{mg}$ daily versus $100 \mathrm{mg}$ daily of aspirin.

- Safety and efficacy of statins in pregnant women with APS who develop pre-eclampsia despite treatment with LDA and heparin.

aPL, antiphospholipid antibody; APS, antiphospholipid syndrome; HCQ, hydroxychloroquine; INR, international normalised ratio; LDA, low-dose aspirin; SLE, systemic lupus erythematosus; VKA, vitamin K antagonist.

currently used treatments and answer questions about controversial issues and new potential therapies (box 2).

The cost and availability of suggested treatments are not a barrier to implementation of these recommendations, with the exception of IVIG and plasmapheresis. However, both treatments were recommended as first-line treatment only in CAPS which occurs in less than $1 \%$ of patients, while B cell depletion 
and complement inhibitors may be considered in refractory cases of CAPS. Implementation into clinical practice can be facilitated by the dissemination of the recommendations using online media, by presentations in national and international congresses, development of workshops in meetings of different specialties involved in APS management, or educational lectures for healthcare providers in referral hospitals.

Better understanding of the pathophysiological mechanisms of APS will help to identify new therapeutic targets, and a balance between anticoagulation and immunomodulatory drugs for different APS manifestations. In addition, studies that examine homogeneous patient groups can better evaluate the efficacy and safety of the currently available and new treatments. When sufficient new information will be available, an update of the current recommendations will take place. The task force members believe that these recommendations will help to improve the quality of care in patients with APS and foster future research by highlighting evidence gaps.

\section{Author affiliations}

First Department of Propaedeutic Internal Medicine, Joint Rheumatology program, National and Kapodistrian University of Athens, Athens, Greece

${ }^{2}$ Department of Clinical and Experimental Sciences, University of Brescia, Brescia, Italy

${ }^{3}$ Department of Rheumatology and Clinical Immunology, University Medical Center Utrecht, Utrecht, The Netherlands

${ }^{4}$ Sorbonne University, French National Center for SLE and Aps, Service de Medecine Interne 2, InstitutE3M, Pitié Salpétrière, Paris, France

${ }^{5}$ Autoimmune Diseases, Hospital Clinic, IDIBAPS, University of Barcelona, Barcelona, Spain

${ }^{6}$ Centre de référence maladies auto-immunes et systémiques rares de l'île deFrance, Cochin Hospital, Université Paris Descartes-Sorbonne Paris Cité;INSERM U 1153,

CRESS, Paris, France

${ }^{7}$ Rheumatology Department, Clinica Universidad de Navarra, Madrid, Spain

${ }^{8}$ Department of Med/Rheumatology and Clinical Immunology, Charite University Hospital, Berlin, Germany

${ }^{9}$ Obstetrics and Gynecology Department and Systemic Diseases Research Unit, Vall d'Hebron Research Institute-VHIR, Barcelona, Spain

${ }^{10}$ School of Sport and Exercise Sciences, University of Kent, Chatham, UK

${ }^{11}$ Rheumatology Department, Dubai Hospital, Dubai, United Arab Emirates

${ }^{12}$ EULAR PARE Patient Research Partner, London, UK

${ }^{13}$ EULAR PARE Patient Research Partner, Rome, Italy

${ }^{14} \mathrm{MaACR}$, Immunorheumatology Research Laboratory, Istituto Auxologico Italiano, Milan, Italy

${ }^{15}$ Department of Clinical and Experimental Medicine, University of Pisa, Pisa, Italy

${ }^{16}$ Department of Cardiac Thoracic and Vascular Sciences and Public Health, University of Padova, Padua, Italy

${ }^{17}$ Department of Obstetrics and Gynaecology, University Hospital of Bern, Inselspital, Bern, Switzerland

${ }^{18}$ Autoimmune Diseases Unit, Hospital Universitario Cruces, Barakaldo, Spain

${ }^{19}$ Zabludowicz Center for Autoimmune Diseases, Sheba Medical Center, Tel-Aviv

University, Israel

${ }^{20}$ Bezhaniijska Kosa, Belgrade University, Belgrade, Serbia

${ }^{21}$ Department of Medicine, Solna, Rheumatology Unit, Karolinska Institutet, Karolinska University Hospital, Stockholm, Sweden

${ }^{22}$ Vascular Medicine Division and Regional Competence Center for Rare Vascular and Systemic Autoimmune Diseases and Vascular Medicine Division, Nancy University Hospital, INSERM UMR-S 1116 University of Lorraine, Nancy, France

${ }^{23}$ Intramural Research Program, National Institute of Arthritis and Musculoskeletal and Skin Diseases, National Institutes of Health, Bethesda, Maryland, USA

Correction notice This article has been corrected since it published Online First. The discussion section has been amended and the title in table one.

Contributors Full-text review, data abstraction and risk of bias assessments were performed by $\mathrm{LA}$ and $\mathrm{ML}$, and independently double-checked by MGT and MMW. MMW supervised the methodology of the SLR and prepared the evidence report. MGT and AT prepared the first draft of recommendations, and all authors have participated in the discussion and formulation of recommendations. MGT supervised the project and drafted the manuscript. All authors reviewed the manuscript and approved its final version.

Funding This project was funded by the European League Against Rheumatism. MMW was funded by the Intramural Research Program, National Institute of Arthritis and Musculoskeletal and Skin Diseases, NIH.
Competing interests None declared.

Patient consent for publication Not required.

Provenance and peer review Not commissioned; externally peer reviewed.

\section{REFERENCES}

1 Miyakis S, Lockshin MD, Atsumi T, et al. International consensus statement on an update of the classification criteria for definite antiphospholipid syndrome (APS). J Thromb Haemost 2006:4:295-306.

2 Cervera R, Serrano R, Pons-Estel GJ, et al. Morbidity and mortality in the antiphospholipid syndrome during a 10-year period: a multicentre prospective study of 1000 patients. Ann Rheum Dis 2015;74:1011-8.

3 Ruiz-Irastorza G, Cuadrado MJ, Ruiz-Arruza I, et al. Evidence-based recommendations for the prevention and long-term management of thrombosis in antiphospholipid antibody-positive patients: report of a task Force at the 13th International Congress on antiphospholipid antibodies. Lupus 2011;20:206-18.

4 Garcia D, Erkan D. Diagnosis and management of the antiphospholipid syndrome. N Engl J Med 2018;378:2010-21.

5 van der Heijde D, Aletaha D, Carmona L, et al. 2014 update of the EULAR standardised operating procedures for EULAR-endorsed recommendations. Ann Rheum Dis 2015;74:8-13

6 Brouwers MC, Kho ME, Browman GP, et al. Agree II: advancing Guideline Development, reporting and evaluation in health care. CMAJ 2010;182:E83 9-E842.

7 Pengo V, Denas G, Zoppellaro G, et al. Rivaroxaban vs warfarin in high-risk patients with antiphospholipid syndrome. Blood 2018;132:1365-71.

8 OCEBM Levels of Evidence Working Group. The Oxford levels of evidence 2: Oxford centre for evidence-based medicine. Available: http://www. cebm. net/ index. aspx? $0=5653$

9 Tektonidou MG, Andreoli L, Limper M, et al. Management of thrombotic and obstetric antiphospholipid syndrome: a systematic literature review Informing the EULAR recommendations for the management of antiphospholipid syndrome in adults. RMD Open.

10 Chighizola CB, Andreoli L, Gerosa M, et al. The treatment of anti-phospholipid syndrome: a comprehensive clinical approach. J Autoimmun 2018;90:1-27.

11 Otomo K, Atsumi T, Amengual O, et al. Efficacy of the antiphospholipid score for the diagnosis of antiphospholipid syndrome and its predictive value for thrombotic events. Arthritis Rheum 2012;64:504-12.

12 Sciascia S, Sanna G, Murru V, et al. GAPSS: the global anti-phospholipid syndrome score. Rheumatology 2013;52:1397-403.

13 Andreoli L, Bertsias GK, Agmon-Levin N, et al. EULAR recommendations for women's health and the management of family planning, assisted reproduction, pregnancy and menopause in patients with systemic lupus erythematosus and/or antiphospholipid syndrome. Ann Rheum Dis 2017;76:476-85.

14 Arnaud L, Mathian A, Ruffatti A, et al. Efficacy of aspirin for the primary prevention of thrombosis in patients with antiphospholipid antibodies: an international and collaborative meta-analysis. Autoimmun Rev 2014;13:281-91.

15 Arnaud L, Mathian A, Devilliers $\mathrm{H}$, et al. Patient-level analysis of five international cohorts further confirms the efficacy of aspirin for the primary prevention of thrombosis in patients with antiphospholipid antibodies. Autoimmun Rev 2015;14:192-200

16 Tarr T, Lakos G, Bhattoa HP, et al. Analysis of risk factors for the development of thrombotic complications in antiphospholipid antibody positive lupus patients. Lupus 2007:16:39-45.

17 Tektonidou MG, Laskari K, Panagiotakos DB, et al. Risk factors for thrombosis and primary thrombosis prevention in patients with systemic lupus erythematosus with or without antiphospholipid antibodies. Arthritis Rheum 2009;61:29-36.

18 Erkan D, Merrill JT, Yazici Y, et al. High thrombosis rate after fetal loss in antiphospholipid syndrome: effective prophylaxis with aspirin. Arthritis Rheum 2001:44:1466-7.

19 Forastiero R, Martinuzzo M, Pombo G, et al. A prospective study of antibodies to beta2-glycoprotein I and prothrombin, and risk of thrombosis. J Thromb Haemost 2005:3:1231-8.

20 Ruffatti A, Del Ross T, Ciprian M, et al. Risk factors for a first thrombotic event in antiphospholipid antibody carriers: a prospective multicentre follow-up study. Ann Rheum Dis 2011;70:1083-6.

21 Crowther MA, Ginsberg JS, Julian J, et al. A comparison of two intensities of warfarin for the prevention of recurrent thrombosis in patients with the antiphospholipid antibody syndrome. N Engl J Med 2003:349:1133-8.

22 Finazzi G, Marchioli R, Brancaccio V, et al. A randomized clinical trial of high-intensity warfarin vs. conventional antithrombotic therapy for the prevention of recurrent thrombosis in patients with the antiphospholipid syndrome (WAPS). J Thromb Haemost 2005:3:848-53.

23 Rosove MH, Brewer PM. Antiphospholipid thrombosis: clinical course after the first thrombotic event in 70 patients. Ann Intern Med 1992;117:303-8.

24 Khamashta MA, Cuadrado MJ, Mujic F, et al. The management of thrombosis in the antiphospholipid-antibody syndrome. N Eng/ J Med 1995;332:993-7. 
25 Ames PRJ, Ciampa A, Margaglione M, et al. Bleeding and re-thrombosis in primary antiphospholipid syndrome on oral anticoagulation: an 8-year longitudinal comparison with mitral valve replacement and inherited thrombophilia. Thromb Haemost 2005;93:694-9.

26 Goldhaber SZ, Eriksson H, Kakkar A, et al. Efficacy of dabigatran versus warfarin in patients with acute venous thromboembolism in the presence of thrombophilia: findings from RE-COVER ${ }^{\circledR}$, RE-COVERTM $\|$, and RE-MEDYTM. Vasc Med 2016:21:506-14.

27 Cohen H, Hunt BJ, Efthymiou M, et al. Rivaroxaban versus warfarin to treat patients with thrombotic antiphospholipid syndrome, with or without systemic lupus erythematosus (RAPS): a randomised, controlled, open-label, phase 2/3, non-inferiority trial. Lancet Haematol 2016:3:e426-36.

28 Schulman S, Svenungsson E, Granqvist S. Anticardiolipin antibodies predict early recurrence of thromboembolism and death among patients with venous thromboembolism following anticoagulant therapy. Duration of Anticoagulation Study Group. Am J Med 1998;104:332-8.

29 Ginsberg JS, Wells PS, Brill-Edwards P, et al. Antiphospholipid Antibodies and venous thromboembolism. Blood 1995;86:3685-91.

30 Kearon C, Akl EA, Ornelas J, et al. Antithrombotic therapy for VTe disease: chest guideline and expert panel report. Chest 2016;149:315-52.

31 Verro P, Levine SR, Tietjen GE. Cerebrovascular ischemic events with high positive anticardiolipin antibodies. Stroke 1998;29:2245-53.

32 Wang C-R, Liu M-F. Rituximab usage in systemic lupus erythematosus-associated antiphospholipid syndrome: a single-center experience. Semin Arthritis Rheum 2016;46:102-8

33 Levine SR, Brey RL, Tilley BC, et al. Antiphospholipid Antibodies and subsequent thrombo-occlusive events in patients with ischemic stroke. JAMA 2004;291:576-84.

34 Tektonidou MG, loannidis JP, Boki KA, et al. Prognostic factors and clustering of serious clinical outcomes in antiphospholipid syndrome. QJM 2000:93:523-30.

35 Ruiz-Irastorza G, Hunt BJ, Khamashta MA. A systematic review of secondary thromboprophylaxis in patients with antiphospholipid antibodies. Arthritis Rheum 2007; 57:1487-95.

36 Krnic-Barrie S, O'Connor CR, Looney SW, et al. A retrospective review of 61 patients with antiphospholipid syndrome. Analysis of factors influencing recurrent thrombosis. Arch Intern Med 1997:157:2101-8.

37 Okuma H, Kitagawa Y, Yasuda T, et al. Comparison between single antiplatelet therapy and combination of antiplatelet and anticoagulation therapy for secondary prevention in ischemic stroke patients with antiphospholipid syndrome. Int J Med Sci 2009;7:15-18.

38 Dufrost V, Risse J, Reshetnyak T, et al. Increased risk of thrombosis in antiphospholipid syndrome patients treated with direct oral anticoagulants. Results from an international patient-level data meta-analysis. Autoimmun Rev 2018;17:1011-21.

39 Uthman I, Noureldine MHA, Ruiz-Irastorza G, et al. Management of antiphospholipid syndrome. Ann Rheum Dis 2019;78:155-61.

40 Erkan D, Unlu O, Sciascia S, et al. Hydroxychloroquine in the primary thrombosis prophylaxis of antiphospholipid antibody positive patients without systemic autoimmune disease. Lupus 2018:27:399-406.

41 Andrade D, Tektonidou M. Emerging therapies in antiphospholipid syndrome. Curr Rheumatol Rep 2016;18.

42 Kaaja R, Julkunen H, Viinikka L, et al. Production of prostacyclin and thromboxane in lupus pregnancies: effect of Small dose of aspirin. Obstet Gynecol 1993;81:229-31.

43 Cowchock S, Reece EA. Do low-risk pregnant women with antiphospholipid antibodies need to be treated? organizing group of the antiphospholipid antibody treatment trial. Am J Obstet Gynecol 1997;176:1099-100.

44 Kahwa EK, Sargeant LA, McCaw-Binns A, et al. Anticardiolipin antibodies in Jamaican primiparae. J Obstet Gynaecol 2006;26:122-6.

45 Del Ross T, Ruffatti A, Visentin MS, et al. Treatment of 139 pregnancies in antiphospholipid-positive women not fulfilling criteria for antiphospholipid syndrome: a retrospective study. J Rheumatol 2013;40:425-9.

46 Rai R, Cohen H, Dave M, et al. Randomised controlled trial of aspirin and aspirin plus heparin in pregnant women with recurrent miscarriage associated with phospholipid antibodies (or antiphospholipid antibodies). BMJ 1997:314:253-7.

47 Lima F, Khamashta MA, Buchanan NM, et al. A study of sixty pregnancies in patients with the antiphospholipid syndrome. Clin Exp Rheumatol 1996:14:131-6.
48 Muñoz-Rodriguez FJ, Font J, Cervera R, et al. Clinical study and follow-up of 100 patients with the antiphospholipid syndrome. Semin Arthritis Rheum 1999;29:182-90.

49 Naru T, Khan RS, Ali R. Pregnancy outcome in women with antiphospholipid syndrome on low-dose aspirin and heparin: a retrospective study. East Mediterr Health $J$ 2010;16:308-12.

50 Cohn DM, Goddijn M, Middeldorp S, et al. Recurrent miscarriage and antiphospholipid antibodies: prognosis of subsequent pregnancy. J Thromb Haemost 2010;8:2208-13.

51 Mohamed KAA, Saad AS. Enoxaparin and aspirin therapy for recurrent pregnancy loss due to anti-phospholipid syndrome (APS). Middle East Fertil Soc J 2014;19:176-82.

52 Ruffatti A, Salvan E, Del Ross T, et al. Treatment strategies and pregnancy outcomes in antiphospholipid syndrome patients with thrombosis and triple antiphospholipid positivity. a European multicentre retrospective study. Thromb Haemost 2014;112:727-35.

53 Franklin RD, Kutteh WH. Antiphospholipid antibodies (APA) and recurrent pregnancy loss: treating a unique APA positive population. Hum Reprod 2002;17:2981-5.

54 Laskin CA, Spitzer KA, Clark CA, et al. Low molecular weight heparin and aspirin for recurrent pregnancy loss: results from the randomized, controlled HepASA trial. J Rheumatol 2009;36:279-87.

55 van Hoorn ME, Hague WM, van Pampus MG, et al. Low-molecular-weight heparin and aspirin in the prevention of recurrent early-onset pre-eclampsia in women with antiphospholipid antibodies: the FRUIT-RCT. Eur J Obstet Gynecol Reprod Biol 2016;197:168-73

56 Mekinian A, Lazzaroni MG, Kuzenko A, et al. The efficacy of hydroxychloroquine for obstetrical outcome in anti-phospholipid syndrome: data from a European multicenter retrospective study. Autoimmun Rev 2015;14:498-502.

57 Bramham K, Thomas M, Nelson-Piercy C, et al. First-trimester low-dose prednisolone in refractory antiphospholipid antibody-related pregnancy loss. Blood 2011;117:6948-51.

58 Diejomaoh MF, Al-Azemi MM, Bandar A, et al. A favorable outcome of pregnancies in women with primary and secondary recurrent pregnancy loss associated with antiphospholipid syndrome. Arch Gynecol Obstet 2002;266:61-6.

59 Jeremic K, Pervulov M, Gojnic M, et al. Comparison of two therapeutic protocols in patients with antiphospholipid Antibodies and recurrent miscarriages. Vojnosanit Pregl 2005:62:435-9.

60 Heilmann L, Schorch M, Hahn T, et al. Pregnancy outcome in women with antiphospholipid antibodies: report on a retrospective study. Semin Thromb Hemost 2008:34:794-802.

61 Stone S, Hunt BJ, Khamashta MA, et al. Primary antiphospholipid syndrome in pregnancy: an analysis of outcome in a cohort of 33 women treated with a rigorous protocol. J Thromb Haemost 2005;3:243-5.

62 Bramham K, Hunt BJ, Germain S, et al. Pregnancy outcome in different clinical phenotypes of antiphospholipid syndrome. Lupus 2010;19:58-64.

63 Fischer-Betz R, Specker C, Brinks R, et al. Pregnancy outcome in patients with antiphospholipid syndrome after cerebral ischaemic events: an observational study. Lupus 2012;21:1183-9.

64 Saccone G, Berghella V, Maruotti GM, et al. Antiphospholipid antibody profile based obstetric outcomes of primary antiphospholipid syndrome: the PREGNANTS study. Am J Obstet Gynecol 2017;216:525.e1-525.e12.

65 de Jesús GR, Sciascia S, Andrade D, et al. Factors associated with first thrombosis in patients presenting with obstetric antiphospholipid syndrome (APS) in the APS Alliance for clinical trials and international networking clinical database and Repository: a retrospective study. BJOG 2019;126.

66 Bates SM, Middeldorp S, Rodger M, et al. Guidance for the treatment and prevention of obstetric-associated venous thromboembolism. J Thromb Thrombolysis 2016;41:92-128

67 Cervera R, Rodríguez-Pintó I, Colafrancesco S, et al. 14th International Congress on antiphospholipid antibodies Task Force report on catastrophic antiphospholipid syndrome. Autoimmun Rev 2014:13:699-707.

68 Cervera R, Tektonidou MG, Espinosa G, et al. Task Force on catastrophic antiphospholipid syndrome (APS) and Non-criteria APS manifestations (I): catastrophic APS, APS nephropathy and heart valve lesions. Lupus 2011;20:165-73.

69 Legault K, Schunemann H, Hillis C, et al. McMaster RARE-Best practices clinical practice guideline on diagnosis and management of the catastrophic antiphospholipid syndrome. J Thromb Haemost 201810.1111/jth. 14192. [Epub ahead of print: 07 Jun 2018]. 